\title{
The magnetic moment of the muon worries theorists
}

Francis J.M. Farley

$T$ ast February the Brookhaven muon $(g-2)$ collaboration [1] $L$ announced a new meaurement of the muon $g$-factor, disagreeing with theory by 2.6 standard deviations. Either some term in the theory has been wrongly calculated or this could be the first sign of physics beyond the standard model. To see why this matters let us look back at the electron.

The magnetic moment of the electron is $g$ times the natural unit which is its angular momentum $h / 4 \pi$ multiplied by the Larmor ratio $e / 2 m c$. Dirac theory predicted $g=2$ but we now realise that this is modified by quantum fluctuations. In quantum field theory the electron is surrounded by a thin soup of evanescent particles which wink in and out of existence in fractions of a second. This soup is slightly magnetic so it increases the magnetic moment to $g=2(1+a)$. The small correction a (about $0.12 \%$ ), called the anomalous moment, but often referred to as " $\mathrm{g}-2$ " is a pure quantum effect entirely due to the soup of virtual particles around the electron. Its detection and measurement with gradually increasing accuracy inspired the theory of quantum electrodynamics (QED) and the present spectacular agreement [2] with calculation to 24 parts per billion ( $\mathrm{pPb}$ ) in a is one of the main pillars of the theory. (The measurement is more accurate, but independent knowledge of the fine structure constant $\alpha$ limits the comparison).

The muon is 206 times heavier than the electron so its magnetic moment is 206 times smaller, but the virtual particles in the quantum soup can be more massive. As a result the anomalous moment is 40,000 times more sensitive to undiscovered particles and new physics at short distances,

$$
\Delta a / a \sim(m / M)^{2}
$$

where $m$ is the mass of the muon (or electron) and $M$ is the mass scale of some new process. The present accuracy in the muon anomaly $a_{\mu}$ is 1.3 parts per million (ppm). In comparing with theory the error is $\mathbf{5 0}$ times larger than for the electron, but this makes it 800 times more sensitive to new effects. The agreement to $4 \mathrm{ppm}$ must be regarded as the best test of the theory, but there is a small discrepancy that needs to be explained.

How can one measure $a_{\mu}$ ? When longitundinally polarised muons are trapped in a magnetic field $B$ the orbit angular frequency is

$$
\omega_{c}=e B / m c
$$

where $e$ is its electric charge, $m$ its mass and $c$ the velocity of light, while the spin frequency is

$$
\omega_{s}=g(e / 2 m c) B
$$

If $g=2$ exactly these two frequencies are identical and the spin is always aligned with the velocity vector. But if $g>2$, it turns faster and the angle between spin and momentum increases at angular frequency

$$
\omega_{a}=(g / 2-1)(e B / m c)=a_{\mu}(e B / m c)
$$

It would be convenient to calibrate the magnetic field by measuring muon precession at rest, combining equations (3) and (4) to give

$$
\omega_{a} / \omega_{s}=a_{\mu} /\left(1+a_{\mu}\right)
$$

Then $(e / m c)$ for the muon need not be known. In practice one measures the field in terms of the proton precession (NMR) frequency $\omega_{p}$ and converts to ws using the ratio $\lambda=\omega_{s} / \omega_{p}=\mu_{\mu} / \mu_{p}$ where $\mu_{\mu}$ and $\mu_{p}$ are the magnetic moments of the muon and proton. The value of $\lambda$ is known to $26 \mathrm{ppb}$ from the hyperfine structure of muonium [3].

In summary, one injects polarised muons into a magnetic field calibrated by proton NMR and measures the rate at which the spin turns relative to the momentum; this determines am rather than $g$ so it has come to be called the $(g$-2)-experiment. At low velocities the spin turns once relative to the velocity for every 800 turns in the field, so a good measurement requires many orbits. In a reasonable magnetic field $(1.6 T)$ the $(g-2)$ period (of spin relative to momentum) is $4 \mu \mathrm{s}$, but the muon lifetime is only $2.2 \mu \mathrm{s}$, so there are not many precession cycles to measure. The solution is to use highly relativistic muons so the lifetime is dilated (moving clocks run slow). Fortunately (for more complicated reasons) $\omega_{a}$ is not slowed down and equation.(4) remains valid; in the latest experiments the muons are kept in the field for over $700 \mu$ s givng hundreds of cycles to measure.

In $1956(\mathrm{~g}-2)$ measurements for the electron were in progress and explained by QED; Berestetskii and colleagues derived equation (1) and called for a measurement of $a_{\mu}$ as a far better test of the theory. But in 1956 parity was conserved and muons were unpolarised, so there was no way to do the experiment.

In 1957 parity was violated and the muons from $\pi \rightarrow \mu+v$ decay were found to be longitudinally polarised. In a footnote to their first paper on muon precession Garwin, Lederman and Weinrich [4] invoked the $(g-2)$ formula (4) to deduce that $g=2$ to within $10 \%$. Attempts to store muons in a magnet for many turns were initiated in several laboratories.

In 1960 muons from the CERN cyclotron were focused onto a beryllium blck inside a long bending magnet, fig.1, so that they lost energy and were trapped in the field $[5,6]$. A transverse gradient (high field at the bottom of fig.1, lower field at the top) made the orbits walk to the right, $2 \mathrm{~cm}$ per turn near the beryllium falling to $0.4 \mathrm{~cm}$ per turn in the middle of the magnet. When the muon reached the end it encountered a much steeper gradient, stepped along at $11 \mathrm{~cm}$ per turn, emerged from the field and was stopped in a field free absorber. After an average of $2.2 \mu \mathrm{s}$ it decayed, $\mu^{+} \rightarrow e^{+}+\nu+v_{\mu}$, and the positrons were emitted preferentially in the direction of the muon spin. They were detected by scintillators in front of and behind the absorber so that over many events the average direction of the muon spin could be recorded. Fig. 2 shows the transverse spin angle vs time spent in the magnet; a fit to this data determined $a_{\mu}$ to $0.4 \%$. At the time the result was a surprise, it confirmed QED up to a mass scale of $1 \mathrm{GeV}$ (while the pundits including R.P. Feynman had been anticipating a discrepancy) and it gave no sign of a new field postulated to explain the muon mass. Now the muon was accepted as a heavy electron and 
nothing more, and this was confirmed by experiments at high energy, muon pair production by photons and the trident process (muon pairs produced by high energy muons), which showed that the muon obeys Fermi-Dirac statistics.

In 1962 the CERN proton synchrotron (PS) was running and high energy muons were available. Could we use them for $(g-2)$, dilating the lifetime to get more precession cycles? How to inject into a large ring magnet, and how to follow the spin direction vs time? On this occasion Nature was miraculously on our side! It is easy to inject, just put a target in the ring and hit it with high energy protons. Pions are produced and start to turn around the ring. In a few turns they decay to muons; the change of momentum in the decay shrinks the orbit so some muons forever miss the proton target and are permanently stored. They come from forward decay and are highly polarised.

When the muon decays some energy is lost to neutrinos, so the decay electron is bent to a smaller radius, emerges on the inside of the ring and hits a counter. By selecting large pulses from the lead/scintillator sandwich one can select high energy electrons and these must come from forward decay in the muon rest frame. Selecting energy in the laboratory selects angle in the moving frame. Therefore, as the muon spin rotates, the number of detected electrons is modulated. One sees an exponential decay with the dilated muon lifetime (27 $\mu s$ for $1.2 \mathrm{GeV}$ muons) with the ( $g$-2) precession superimposed. Read off the frequency and you can measure $\omega_{a}$. Fig. 3 the precession curve [7] obtained in 1968.

To ensure vertical focusing, essential for storing muons for many turns, the field of the ring magnet had a radial gradient of $54 \mathrm{ppm} / \mathrm{mm}$. So to determine the mean field and calculate $a_{\mu}$ one needed to know the radius of the stored muons. Fortunately the muons were bunched at injection and at early times the counting rate was modulated by the orbit frequency, (fig.3, lowest curve). From the record, the muon radius could be calculated to $\pm 3 \mathrm{~mm}$. This experiment initially disagreed with theory by $(450 \pm 270) \mathrm{ppm}$, stimulating a reexamination by theorists of some $(\alpha / \pi)^{3}$-terms in the QED expansion, connected theoretically with the scattering of light by light which has never been observed. They found a surprisingly large coefficient of 18.6, which brought the theory into agreement with the data.

In 1969 the main obstacle to further improvement was the radial magnetic gradient needed for vertical focusing. It was considered impossible to know the radius of the muons to better than 1 $\mathrm{mm}$. So in the third CERN experiment the magnet had a uniform magnetic field with vertical focusing provided by electric quadrupoles. Positive plates above and below the orbit repelled the $\mu^{+}$ back towards the centre. One can show that the effect on the orbits is equivalent to a weak focusing system with magnetic gradient and surprisingly about $20 \mathrm{kV}$ is sufficient to focus $3 \mathrm{GeV}$ muons.

It is the vertical component of the electric field that does the focusing, but inevitably it is associated with a horizontal electric field that varies with radius. In general a radial electric field affects the spin motion, so it is still necessary to know the muon radius. Nothing is gained - unless one operates at a carefully chosen energy! If however $\gamma=E / m c^{2}=\sqrt{ }(1+1 / a)=29.3$ a radial electric field does not change the $(g-2)$ frequency; then one can use a uniform magnetic field with vertical focusing provided by electric quadrupoles; and the $(\mathrm{g}-2)$ frequency will be the same all over the aperture. One does not need to know where the muons are! This so-called "magic" energy is $3.096 \mathrm{GeV}$, easily accessible with current accelerators.

In fact a correction is required for muons which are not exactly centred in the aperture; their momentum is not exactly magic and there is some radial electric field. On average this correction (calculated from the radial distribution of muons obtained from the orbit frequency data) is about $0.5 \mathrm{ppm}$.

The third CERN experiment [8] used a $14 \mathrm{~m}$ diameter storage ring operating at the magic energy. To gain beam intensity and

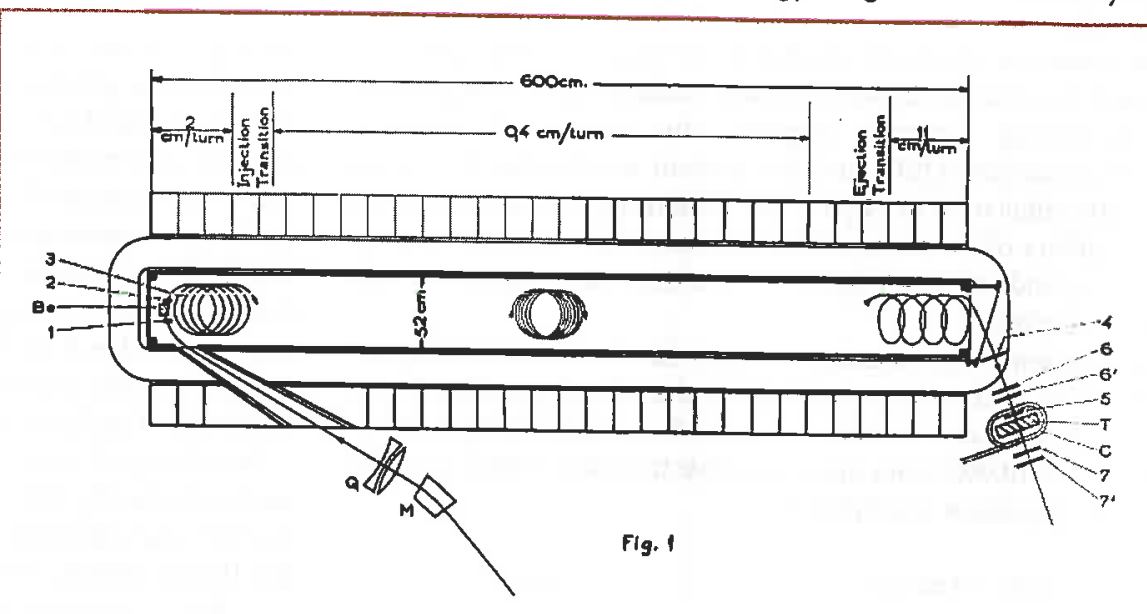

Fig. 1: First muon ( $g$-2) experiment at CERN. Muons from the cyclotron lost energy in the beryllium block and were trapped in the field of the $6 \mathrm{~m}$ long magnet. A weak transverse gradient made the orbits walk to the right and emerge from the field after $200-1200$ turns.

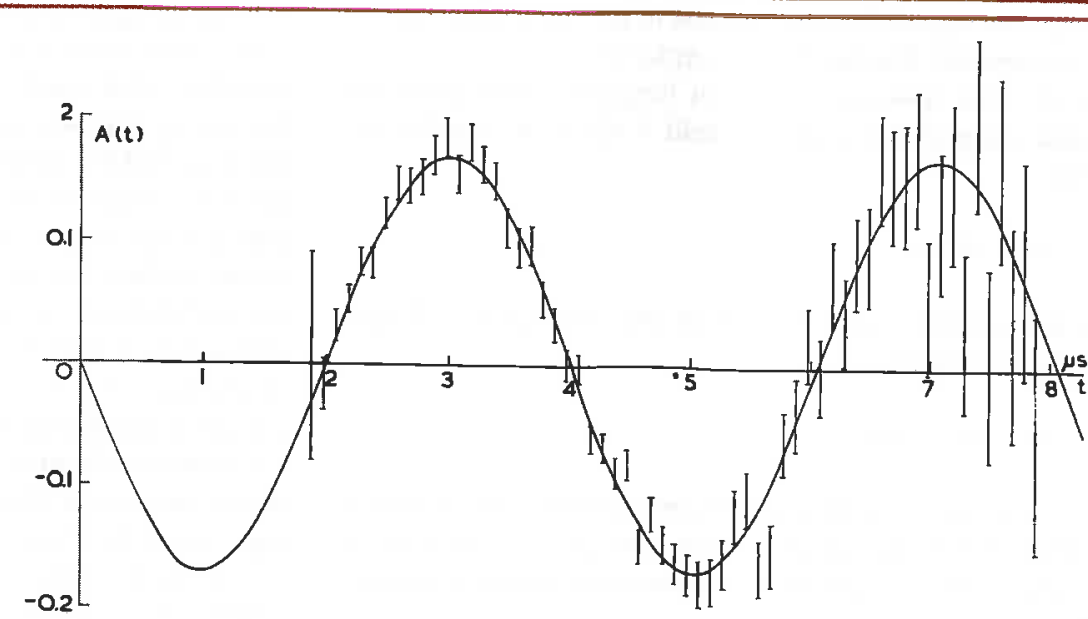

Fig. 2: $1962-(g-2)$ precession in long magnet giving $a_{\mu}$ to $\pm 0.4 \%$. 
reduce background, pions were injected (instead of protons). The magnetic field was stabilised at 40 points by feedback from NMR probes, and was very reproducible. The measurement to $7.3 \mathrm{ppm}$ agreed with theory, and confirmed the existence of virtual hadrons in the quantum soup around the muon, calculated to contribute $58 \mathrm{ppm}$ to the value of $a_{\mu}$. Hadrons are not coupled directly to the muon, but a virtual photon in the soup can produce a virtual quark-antiquark pair. Once this happens the quarks interact strongly with each other, exchanging gluons and all the glory of the strong interaction comes into play, quarkantiquark convert to $\pi^{+} \pi^{-}, \rho, \phi$, and $\omega$ resonances and all sorts of higher states. The equations which govern the strong interactions (quantum chromodynamics, QCD) are presumed known, but no one can solve them for the strongly coupled low energy states which contribute most to $a_{\mu}$. How the effect is estimated is discussed below.

Also present in the soup are the intermediate bosons of the weak interaction, $Z^{0}$ and $W^{ \pm}$, which couple directly to the muon and mediate its decay to $e^{+} v v_{\mu}$. Their effect, calculated from the electroweak theory, is predicted to be 1.3 ppm in $a_{\mu}$.

To see this effect was a primary objective of the new more accurate measurement of muon $(g-2)$ initiated by Vernon Hughes of Yale in 1984. Improvements in the $33 \mathrm{GeV}$ Brookhaven synchrotron (AGS) meant that many more muons could be available so the statistical error could be greatly reduced. $\mathrm{He}$ gradually assembled a large team of collaborators including, in addition to Brookhaven, groups from Boston University, Illinois, Minnesota, Germany, Japan and Russia and some of the old CERN team. It was decided to operate again at the magic energy with electric focusing, using a larger magnet aperture to give a more uniform field. Superconducting coils were used to excite an iron magnet with field $1.45 T$ and orbit diameter $14.22 \mathrm{~m}$, fig. 4. Muons were collected from $\pi-\mu$ decay in a long focusing channel and separated from the undecayed particles by momentum selection. They were then injected into the storage ring and kicked onto the correct orbit with a pulsed magnetic field. Compared to pion injection this is more complex, but gives many more stored muons and a large reduction in background, essential for operation at the high beam intensities now available.

To measure the magnetic field a trolley, carrying NMR probes and a computer, was made to move round the ring inside the vacuum chamber and measure the field along the track of the muons at any moment. A single coaxial cable carried DC power to the electronics and a $62 \mathrm{MHz}$ reference frequency and served to pull the trolley round the ring. It also carried output signals from the computer giving the results of the measurements.

During the muon storage runs the trolley was withdrawn into a special garage, (also under vacuum) and the field was monitored by 350 fixed NMR probes deployed above and below the vacuum chamber. The average field calculated from these fixed probes tracked with the average meaured by the trolley to within $\pm 0.2 \mathrm{ppm}$. A fit to the data taken in 1999, fig.5, and published last February $[1,9]$ gave $a_{\mu}=1,165,920.2 \times 10^{-9}$ with an error of $1.34 \mathrm{ppm}$. Combining with the previous measurements gives the best experimental value $a_{\mu}=1,165,920.3 \times 10^{-9}$ with an error of 1.27 ppm compared to the theoretical prediction $a_{\mu}=1,165,916.0 \times 10^{-9}$ with an error of $0.57 \mathrm{ppm}$ [10]. The discrepancy of $(3.7 \pm 1.4)$ ppm warrants some discussion.

It could be a statistical fluke but that is unlikely. It could be a "harbinger for new physics" [10]. In particular the theory of Supersymmetry [11] (which has many adjustable parameters) predicts a new contribution to am and can easily accommodate our value. In this theory every known boson is matched by a supersymmetric fermion, and vice versa, so many new particles would be there awaiting discovery as soon we can reach sufficient energy; and the theory gets rid of some mathematical infinities that plague the standard model. 


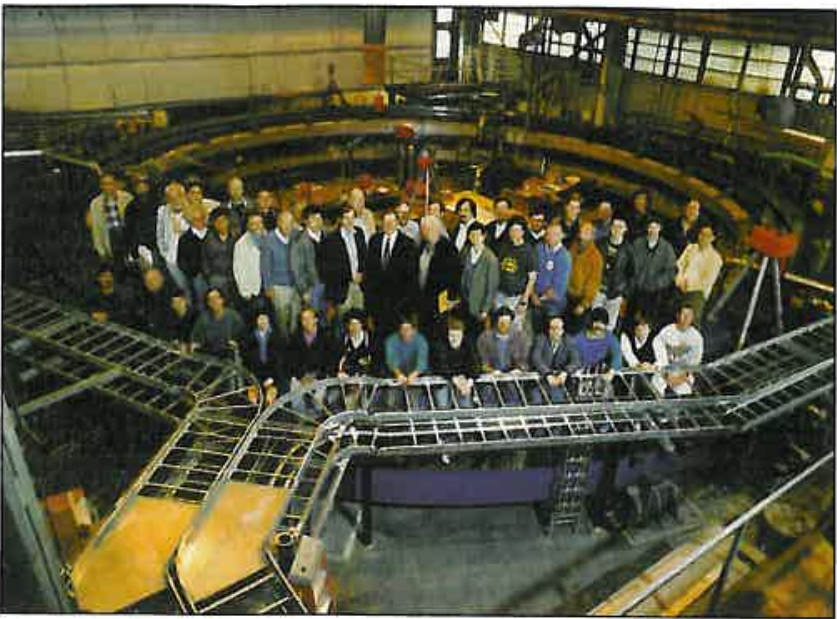

Fig. 4: Brookhaven $14 \mathrm{~m}$ diameter superferric storage ring with some of the $(g-2)$ group.

On the other hand the current standard model (renormalisable electroweak theory, plus quantum chromodynamics) may not have been correctly evaluated. No one doubts the main terms derived from QED and the weak intereaction, but the calculation of the hadronic part of $a_{\mu}$ is complex and in some parts approximate. The main effect (one virtual hadron in the soup converting to a quark-antiquark pair) is related by dispersion theory to the probability of creating hadrons in electron-positron colliding beams, and this has been carefully measured in Novosibirsk and Beijing and discussed by many theorists. There is currently a consensus about this part of $a_{\mu}$ but new experimental data could still change the value. On the other hand there are more complex hadronic processes involving several virtual photons and these contribute to $a_{\mu}$ at the level of $1 \mathrm{ppm}$ and in this area no one is quite sure of the calculation.

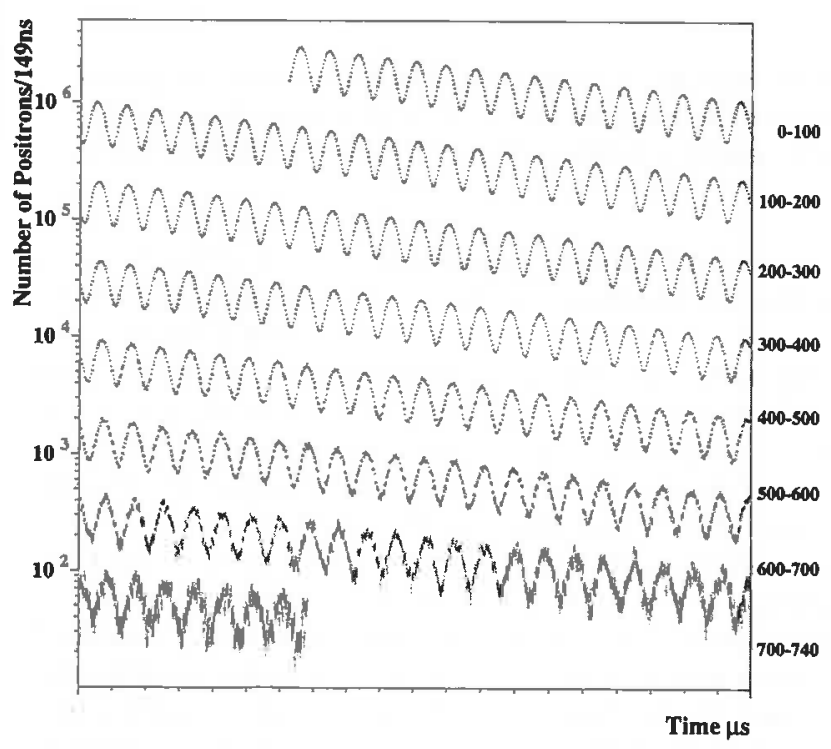

Fig. 5: 1999 muon ( $g-2$ ) precession measured out to $740 \mathrm{~ms}$ at Brookhaven with lifetime dilated to $64 \mu \mathrm{s}$, giving $a_{\mu}$ to \pm 1.3 ppm, $2.6 \sigma$ above theory; it could be a "harbinger for new physics" [10]
So in summary the new muon ( $g$-2) result from Brookhaven cannot at present be explained by the established theory. A more accurate meaurement, based on data taken in the years 2000 and 2001 shoud be available by the end of the year. Meanwhile theorists are looking for flaws in the argument and more measurements on the production of hadrons by $e^{+} e^{-}$colliding beams are underway. If all this fails, Supersymmetry can explain the data, but we would need other experiments to show that the postulated particles can exist in the real world, as well as in the evanescent quantum soup around the muon.

\section{References}

[1] H.N. Brown, G.Bunce, R.M. Carey, P. Cushman, G.T. Danby, P.T Debevec, M. Deile, H. Deng, W. Deninger, S.K. Dhawan, V.P. Druzhinin, L. Duong, E. Efstathiadis, F.J. M. Farley, G.V. Fedotovich, S. Giron, F. Gray, D. Grigoriev, M. Grosse-Perdekamp, A. Grossmann, M.F. Hare, D.W. Hertzog. V.W. Hughes, M. Iwasaki, K. Jungmann, D. Kawall, M. Kawamura, B.I. Khazin, J. Kindem, F. Krienen, I. Kronkvist, R. Larsen, Y.Y. Lee, I. Logashenko, R. McNabb, W. Meng, J. Mi, J.P. Miller, W.M. Morse, D. Nikas, C.J.G. Onderwater, O. Rind, B.L. Roberts, N. Ryskulov, S. Sedykh, Y.K. Semertzidis, Yu.M. Shatunov, E.P. Sichtermann, E. Solodov, M. Sossong, A. Steinmetz, L.R. Sulak, C. Timmermans, A. Trofimov, D. Urner, P. von Walter, D. Warburton, D. Winn, A. Yamamoto and D. Zimmerman, Phys. Rev. Letters 86, 2227 (2001)

[2] T. Kinoshita, Rep. Prog. Phys 59, 1459 (1996)

[3] D.E. Groom et al., Review of Particle Physics, Eur.Phys.J. C15, 1 (2000)

[4] R.L. Garwin, L.M. Lederman and M. Weinrich, Phys. Rev. 105, 1415 (1957)

[5] G. Charpak, F.J.M. Farley, R.L. Garwin, T. Muller, J.C. Sens and A. Zichichi, Nuovo Cimento 37, 1241 (1965)

[6] For a general review of all three CERN experiments, see FJ.M. Farley and E. Picasso, in Quantum Electrodynamics, T. Kinoshita ed., (World Scientific, 1990).

[7] J. Bailey, W. Bartl, G. von Bochmann, R.C.A. Brown, FJ.M. Farley, M. Giesch, H. Jöstlein, S. van der Meer, E. Picasso, and R.W. Williams, Nuovo Cimento A9, 369 (1972)

[8] J. Bailey, K. Borer, F. Combley, H. Drumm, C. Eck, FJ.M. Farley, J.H. Field, W. Flegel, P.M. Hattersley, F. Krienen, F. Lange, G. Lebée, E. McMillan, G. Petrucci, E. Picasso, O. Rúnofsson, W.von Rüden, R.W. Williams and S. Woijcicki, Nucl. Phys. B150, 1 (1979)

[9] http://phyppro1.phy.bnl.gov/g2muon/index.shtml for the Brookhaven ( $g-2$ ) web site

[10] A. Czarnecki and W.J. Marciano, Nucl. Phys. (Proc. Suppl.) B76, 245 (1999); Phys. Rev, D64 (2001)

[11] S. Weinberg, The Quantum Theory of Fields, Vol 3, Cambridge University Press (1999); John R. Gribbin, The Search for Superstrings, Symmetry and the Theory of Everything, Little Brown \& Co (2000)

\section{About the author}

Francis Farley has worked on all the muon $(g-2)$ experiments. He is a Fellow of the Royal Society which awarded him the 1980 Hughes medal and an honorary Fellow of Trinity College Dublin. He spent many years at CERN and is currently a visiting research scientist at Yale. He has contributed to wartime radar, nuclear reactors at Chalk River, cosmic rays in New Zealand, energy extraction from sea waves and cancer therapy with protons beams. He lives in France. 